\title{
On a Certain Class of Generalized Hypergeometric Functions with Finite Monodromy Groups *)
}

\author{
Takao SASAI \\ Tokyo Metropolitan University
}

\section{Introduction.}

In this paper we shall investigate the monodromy group $G$ of generalized hypergeometric equation (say GHGE, for brevity) in the form of Okubo type, its irreducibility conditions, explicit form of its invariant hermitian matrix and so on. As we shall see later, there exists a complex reflection group $\tilde{G}$ induced from $G$ and containing $G$. We shall also study it and determine the case where $\tilde{G}$ is a finite irreducible group. Then $G$ is also finite and the corresponding solutions of GHGE are algebraic functions.

The equation of Okubo type is a system of first order linear differential equations (see (\#) in $§ 1)$. It gives a fine perspective in the theory of Fuchsian equations to consider those in the form of Okubo type. K. Okubo showed in [5] that every Fuchsian equation can be transformed into one of his type and its monodromy group up to conjugations can be obtained by an algebraic computation. In particular, for GHGE, he obtained the monodromy group in the joint work with Takano ([6]) and solved the connection problem ([5], see also [7]). His theory says that $n$-th order Fuchsian equations in general have $n^{2}-3 n+2$ numbers of accessory parameters (see [5]). We may comprehend above $\tilde{G}$ as the monodromy group of such equation, of which the number of accessory parameters takes the special value 0 , and GHGE as a limit of those equations.

For the single higher order GHGE, the monodromy group was obtained by A. H. M. Levelt in his thesis [4]. We may say that it is integral, i.e:, it is a subgroup of $G L\left(n, \mathcal{O}_{K}\right)$ if its parameters are rational numbers, where $\mathcal{O}_{K}$ is the ring of integers of a suitable cyclotomic field $K$. Recently, in their joint work ([1]), F. Beukers and G. Heckman investigated systematically the cases where the group due to Levelt come to be finite. Theorem 4.8 in [1] has a particular importance which was brought by virtue of the above integral property besides their good idea. Moreover they obtained various consequences by studying a reflection subgroup of the monodromy group. The fact is

\footnotetext{
Received November 5, 1991

*) Supported partly by Grant-in-Aid for Scientific Research (No. 63540065), Ministry of Education.
} 
that, as we shall see later (Lemma 4.5), the two groups due to Levelt and Okubo-Takano are conjugate to each other in $G L(n, C)$. In Proposition 4.6 we shall also state a relation between $G$ and $\tilde{G}$ on the finiteness which was pointed out by $H$. Nakajima.

In case the order of GHGE is equal to 2, it is just Gauss' hypergeometric equation and $\tilde{G}$ coincides with $G$. The determination of finite monodromy groups in this case was already done by $\mathbf{H}$. A. Schwarz which, together with [12], gives a motivation of our present work. Thus we only consider the case where the order of GHGE $\geqq 3$. On Schwarz theory we also refer to [3] and [13].

In §1 we shall summarize several known results on GHGE in the form of Okubo type and its monodromy group. We intend to state those with short proofs in self-contained manner. In $\$ 2$ we shall study the irreducibility conditions for GHGE which coincides with those obtained independently by Beukers-Heckman for the single higher order GHGE with a proof quite different from ours. Next we shall construct in $\$ 3$ the invariant hermitian matrix explicitly under a suitable condition so that its signature will be computed directly. Those results are useful when we study discrete and arithmetic group of GHGE in the future. Main result on the structure of $\widetilde{G}$ and $G$ will be summarized in Theorem 4.3 in $\$ 4$. Though the groups stated here are part of those obtained by Beukers-Heckman, yet our elementary method is applicable to wider class of Fuchsian equations. The results due to G. C. Shephard and J. A. Todd ([10]), and T. A. Springer ([11]) on complex reflection groups play important roles for those investigations. In a certain stage of computations of matrices we used the computer algebra system MACSYMA on DEC VAX-11/750.

Finally I wish to thank to my colleagues, Professor Haruhisa Nakajima for his valuable suggestions on reflection groups, and Professor Teruo Hikita who guided me to computer sciences, especially, to MACSYMA.

\section{§1. Generalized hypergeometric equations in the form of Okubo type and its monodromy group $\boldsymbol{G}$.}

Let $S$ be the Riemann sphere. We consider the following system of linear ordinary differential equations of first order on $S$ which we call of Okubo type;

$$
(t I-B) \frac{d x}{d t}=A x,
$$

where $t$ is a complex variable on $S, x={ }^{t}\left(x_{1}, \cdots, x_{n}\right)$ is a column $n$-vector, $I$ is the $n$ by $\boldsymbol{n}$ unit matrix and, $A$ and $B$ are $n$ by $n$ constant matrices.

Definition 1.1. The equation of Okubo type (\#) is said to be a generalized hypergeometric equation if $B$ is the diagonal matrix 


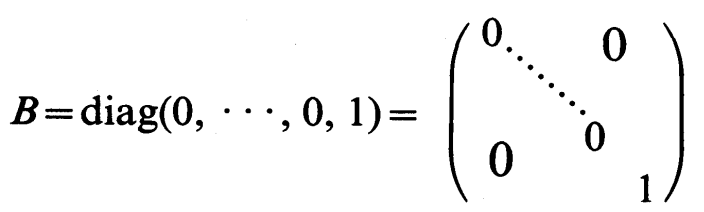

and

$$
A=\left(\begin{array}{ccc|c}
-a_{1} & \ddots & 0 & 1 \\
& \ddots & & \\
0 & \ddots & & \vdots \\
& & -a_{n-1} & 1 \\
\hline b_{1} & \cdots & b_{n-1} & -a_{n}
\end{array}\right)
$$

which has $n$ distinct eigenvalues $-\rho_{1}, \cdots,-\rho_{n}$ (cf. [7], §1).

Throughout this paper we consider (\#) satisfying the following assumption in addition to Definition 1.1.

(A) None of the quantities $a_{i}(i=1,2, \cdots, n), a_{j}-a_{k}(j \neq k ; j, k=1, \cdots, n-1)$ and $\rho_{l}-\rho_{m}(l \neq m ; l, m=1, \cdots, n)$ is an integer. Moreover each $\rho_{l}$ is not a positive integer. Consequently there is no logarithmic solution.

The system (\#) has three regular singular points 0,1 and $\infty$ on $S$. We denote $\exp \left(-2 \pi \sqrt{-1} a_{j}\right)$ and $\exp \left(-2 \pi \sqrt{-1} \rho_{k}\right)$ by $e_{j}$ and $f_{k}$, respectively. The sums and products; $\sum_{k=1}^{n}, \sum_{k=1}^{n-1}, \prod_{k=1}^{n}$ and $\prod_{k=1}^{n-1}$ are also abbreviated to $\sum_{k}, \sum_{k}^{\prime}, \prod_{k}$ and $\prod_{k}^{\prime}$, respectively.

LEMMA 1.2. The characteristic exponents of (\#) are

$$
\begin{array}{ll}
\left(-a_{1}, \cdots,-a_{n-1}, 0\right) & \text { at } t=0, \\
\left(0, \cdots, 0,-a_{n}\right) & \text { at } t=1, \\
\left(\rho_{1}, \rho_{2}, \cdots, \rho_{n}\right) & \text { at } t=\infty .
\end{array}
$$

Riemann-Fuchs relation is simply the invariance of the trace of $A$;

$$
\sum_{k} a_{k}=\sum_{k} \rho_{k} \quad \text { and, consequently, } \prod_{k} e_{k}=\prod_{k} f_{k} \text {. }
$$

LEMMA 1.3. The non-trivial components $b_{j}$ are represented in terms of $a_{k}$ and $\rho_{k}$;

$$
b_{j}=-\frac{\prod_{k}\left(\rho_{k}-a_{j}\right)}{\prod_{k \neq j}^{\prime}\left(a_{k}-a_{j}\right)} \quad(j=1,2, \cdots, n-1) .
$$

Proof. By the definition, $\operatorname{det}(\rho I-A)=\prod_{k}\left(\rho+\rho_{k}\right)$. On the other hand we have 


$$
\operatorname{det}(\rho I-A)=\prod_{k}^{\prime}\left(\rho+a_{k}\right)\left\{\left(\rho+a_{n}\right)-\sum_{l}^{\prime} \frac{b_{l}}{\rho+a_{l}}\right\} .
$$

The lemma follows by setting $\rho=-a_{j}$.

REMARK 1.4. We only note that, if we substitute (1.2) for $b_{j}$ in (\#) and eliminate $x_{1}, \cdots, x_{n-1}$, then $x=x_{n}$ just satisfies the classical GHGE (see Erdélyi [2])

$$
\left[\delta\left(\delta+a_{1}-1\right) \cdots\left(\delta+a_{n-1}-1\right)-t\left(\delta+\rho_{1}\right) \cdots\left(\delta+\rho_{n}\right)\right] x=0,
$$

where $\delta=t(d / d t)$, which has

$$
{ }_{n} F_{n-1}\left(\begin{array}{l}
\rho_{1}, \cdots, \rho_{n} ; t \\
a_{1}, \cdots, a_{n-1}
\end{array}\right)=\sum_{k=0}^{\infty} \frac{\left(\rho_{1}\right)_{k} \cdots\left(\rho_{n}\right)_{k}}{\left(a_{1}\right)_{k} \cdots\left(a_{n-1}\right)_{k} k !} t^{k}
$$

as its particular solution at $t=0$, where $(\alpha)_{k}=\alpha(\alpha+1) \cdots(\alpha+k-1)$. So the system (\#) is equivalent to the equation (b) (for details, see [7], $\S 1$ and also $\S 5$ ).

From Lemma 1.3 , the equation (\#) is determined explicitly by its characteristic exponents: i.e.,

THEOREM 1.5. The system (\#) is accessory parameter free.

THEOREM 1.6 (Gauss-Okubo formula; [5] and [7], Theorem 1). The system (\#) has only $n$ singular solutions defined at 0 and 1 , corresponding to the characteristic exponents $-a_{j}$ and the normalization conditions $g_{j}(0)=\varepsilon_{j}$ :

$$
\left\{\begin{array}{l}
X_{j}(t)=t^{-a_{j}} \sum_{m=0}^{\infty} g_{j}(m) t^{m} \quad(j=1,2, \cdots, n-1), \\
X_{n}(t)=(t-1)^{-a_{n}} \sum_{m=0}^{\infty} g_{n}(m)(t-1)^{m}
\end{array}\right.
$$

where the $j$-th components and the others of the $n$-vector $\varepsilon_{j}$ are 1 and 0 , respectively. Moreover, in any simply connected domain contained in $S^{*}=S \backslash\{0,1, \infty\}$, the Wronskian of these solutions is

$$
\operatorname{det} X=\left(\prod_{k=1}^{n} \frac{\Gamma\left(1-a_{k}\right)}{\Gamma\left(1-\rho_{k}\right)}\right) t^{-\Sigma_{i}^{\prime} a_{l}}(t-1)^{-a_{n}},
$$

where $X$ is the matrix $\left[X_{1}, \cdots, X_{n}\right]$, from which the linear independence of the solutions follows (see (A), in particular, the assumption on $\rho_{l}$ ).

Now we fix a point $o \in S^{*}$. Let $\mu_{0}$ and $\mu_{1}$ be simple loops which start at $\sigma$, go around 0 and 1 , respectively, once in the positive direction and return to 0 . The composition $\mu_{\infty}=\mu_{1} \cdot \mu_{0}$ which is the loop $\mu_{0}$ followed by $\mu_{1}$ is a simple loop surrounding $\infty$ in the negative direction. The loops $\mu_{\alpha}(\alpha=0,1, \infty)$ generate the fundamental group $\pi_{1}\left(S^{*}, 0\right)$ with the fundamental relation $\mu_{\infty}^{-1} \cdot \mu_{1} \cdot \mu_{0}=1$. If the basis $\mathfrak{X}$ for solutions of 
(\#) is continued analytically along $\mu_{\alpha}, \mathfrak{X}$ is transformed into $\mathfrak{X} M_{\alpha}$ where $M_{\alpha} \in G L(n, C)$ is called the circuit matrix around $\alpha$ with respect to $\mathfrak{X}$. Thus a group representation $\varphi$ of $\pi_{1}\left(S^{*}, o\right)$ into $G L(n, C)$ is determined by $\mu_{\alpha} \mapsto M_{\alpha}$. We call the image $\mathscr{G}$ of $\varphi$ the monodromy group of (\#) with respect to $\mathfrak{X}$. If $\mathfrak{X}^{\prime}$ is another basis for solutions of (\#), there exists $T \in G L(n, C)$ which satisfies $\mathfrak{X}^{\prime}=\mathfrak{X} T$. Let $\mathscr{G}^{\prime}$ be the monodromy group with respect to $\mathfrak{X}^{\prime}$. Then we have $\mathscr{G}^{\prime}=T^{-1} \mathscr{G} T$, i.e., the monodromy group of (\#) is determined uniquely up to a conjugation. Now set $\mathfrak{X}=X$ stated in Theorem 1.6. Let $G$ be the corresponding monodromy group. Then we obtain:

LEMMA 1.7. The circuit matrices are represented as $M_{\alpha}=I+C_{\alpha}(\alpha=0,1)$, where

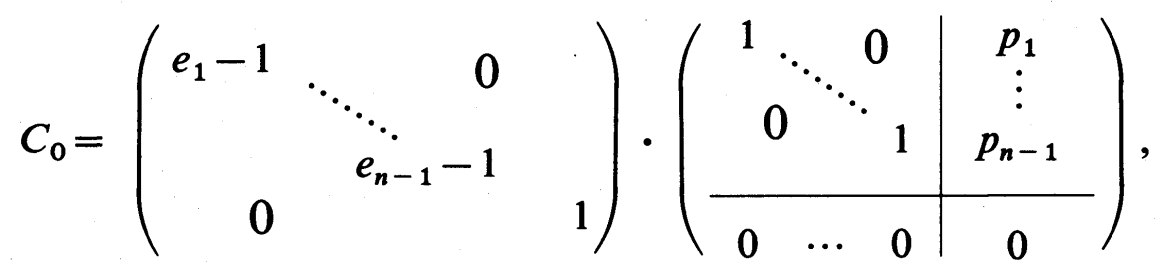

$$
C_{1}=\left(e_{n}-1\right)\left(\frac{0}{q_{1} \cdots q_{n-1} 1}\right) .
$$

The components $p_{j}$ and $q_{j}$ are called the connection coefficients with respect to $X$ which were calculated explicitly by Okubo [5] (see also [7], Theorem 3). However, for observing the group structure of $G$, it is sufficient to obtain only the products $p_{j} q_{j}$ when all of them take non-zero values under which we shall study $G$ from $\S 3$ on (cf. also Theorem 2.3 below). Let $G^{\prime}$ be a group generated by matrices, say $M_{0}^{\prime}$ and $M_{1}^{\prime}$, which are given by substituting $p_{j}^{\prime}$ and $q_{j}^{\prime}$ for $p_{j}$ and $q_{j}$ in (1.3), respectively. We denote it by $G\left(p_{1}^{\prime}, \cdots, p_{n-1}^{\prime} ; q_{1}^{\prime}, \cdots, q_{n-1}\right)$ (for brevity, $\left.G\left(p_{j}^{\prime} ; q_{j}^{\prime}\right)\right)$ if necessary. Then $G=G\left(p_{j} ; q_{j}\right)$.

LEMMA 1.8. Suppose $p_{j} q_{j} \neq 0$ for all $j(1 \leq j \leq n-1)$. For any set $\left\{p_{1}^{\prime}, \cdots, p_{n-1}^{\prime} ; q_{1}^{\prime}\right.$, $\left.\cdots, q_{n-1}^{\prime}\right\}$ there exists a non-singular diagonal matrix $D$ up to a non-zero scalar multiple which satisfies $G\left(p_{j}^{\prime} ; q_{j}^{\prime}\right)=D^{-1} G D$ or, more precisely, $M_{\alpha}^{\prime}=D^{-1} M_{\alpha} D(\alpha=0,1)$, if and only if $p_{k}^{\prime} q_{k}^{\prime}=p_{k} q_{k}$ for all $k(1 \leq k \leq n-1)$.

Proof. We first note that, for any non-singular diagonal matrix $D=$ $\operatorname{diag}\left(\tau_{1}, \cdots, \tau_{n}\right), X \cdot D$ is also a basis for solutions of $(\#)$ and the corresponding monodromy group is $D^{-1} G D$ generated by $D^{-1} M_{\alpha} D=I+D^{-1} C_{\alpha} D$, where 


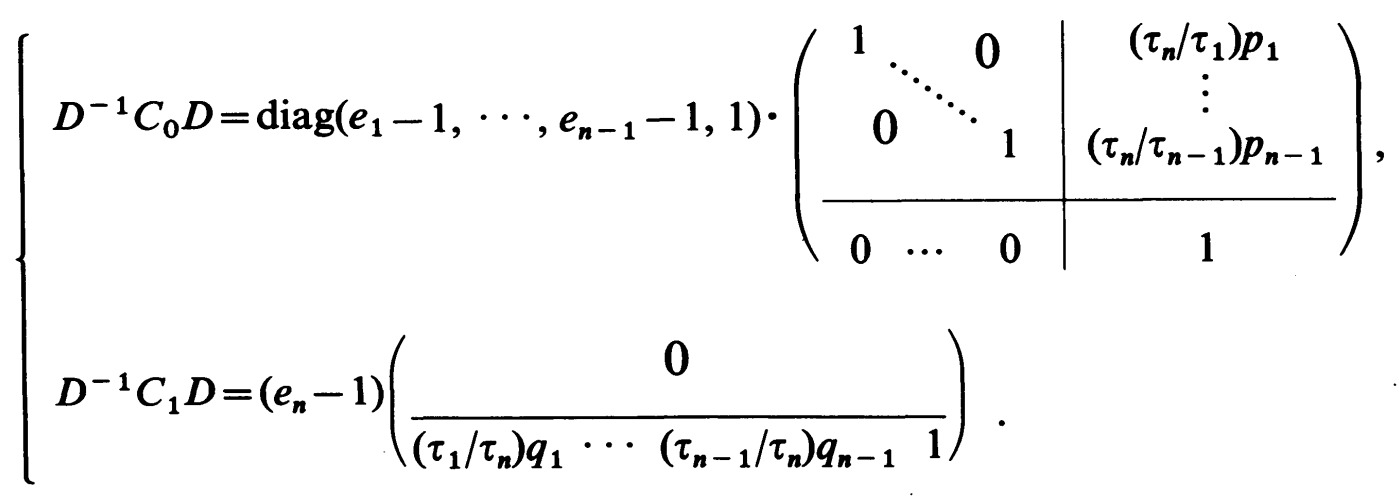

Thus the conditions $M_{\alpha}^{\prime}=D^{-1} M_{\alpha} D$ lead to $p_{j}^{\prime}=\left(\tau_{n} / \tau_{j}\right) p_{j}$ and $q_{j}^{\prime}=\left(\tau_{j} / \tau_{n}\right) q_{j}$ which imply $p_{j}^{\prime} q_{j}^{\prime}=p_{j} q_{j}$. The converse is obvious from (1.4) and relations $\tau_{j} / \tau_{n}=p_{j} / p_{j}^{\prime}=q_{j}^{\prime} / q_{j}$. q.e.d.

REMARK 1.9. From this lemma we can choose appropriate $n-1$ non-zero quantities among $p_{j}$ and $q_{j}$ in (1.3) which take any preassigned values. For example, for an arbitrary choice of $n-1$ non-zero $q_{j}^{\prime}(1 \leq j \leq n-1)$, the group $G^{\prime}=G\left(p_{j}^{\prime} ; q_{j}^{\prime}\right)$ conjugate to $G$ is determined uniquely by taking $p_{j}^{\prime}=p_{j} q_{j} / q_{j}^{\prime}$.

Next we calculate $p_{j} q_{j}$ explicitly. By Lemma 1.2 the eigenvalues of $M_{\infty}=M_{1} \cdot M_{0}$ are $f_{k}(1 \leq k \leq n)$ and, consequently, $\operatorname{det}\left(f I-M_{\infty}\right)=\prod_{k}\left(f-f_{k}\right)$. On the other hand, a direct computation of $\operatorname{det}\left(f I-M_{\infty}\right)$ by (1.3) shows

$$
\prod_{k}\left(f-f_{k}\right)=\left[\left(f-e_{n}\right)-\left(e_{n}-1\right) \sum_{l}^{\prime}\left[\left(e_{l}-1\right)+\frac{e_{l}\left(e_{l}-1\right)}{f-e_{l}}\right] p_{l} q_{l}\right] \cdot \prod_{k}^{\prime}\left(f-e_{k}\right) \text {. }
$$

By setting $f=e_{j}$ we obtain:

THEOREM 1.10 (Okubo-Takano [6], see also [7], Theorem 2).

$$
\begin{aligned}
p_{j} q_{j} & =-\frac{\prod_{k}\left(e_{j}-f_{k}\right)}{e_{j}\left(e_{j}-1\right)\left(e_{n}-1\right) \prod_{k \neq j}^{\prime}\left(e_{j}-e_{k}\right)} \\
& =-\frac{\prod_{k} \sin \pi\left(a_{j}-\rho_{k}\right)}{\sin \pi a_{j} \cdot \sin \pi a_{n} \cdot \prod_{k \neq j}^{\prime} \sin \pi\left(a_{j}-a_{k}\right)} \quad(j=1,2, \cdots, n-1) .
\end{aligned}
$$

The latter relation is implied by (1.1).

\section{§2. Irreducibility conditions for $\boldsymbol{G}$.}

Let $\mathfrak{h}$ be an $n$ by $n$ matrix defined by 


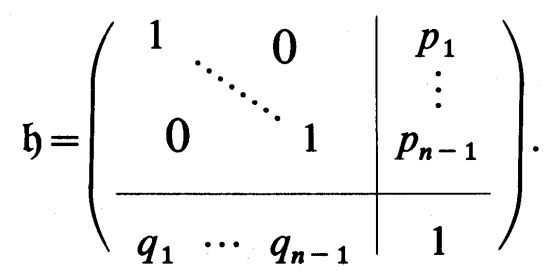

By setting $f=1$ in (1.5) and noting that $\operatorname{det}(\mathfrak{h})=1-\sum_{l}^{\prime} p_{l} q_{l}$, we obtain

$$
\operatorname{det}(\mathfrak{h})=\prod_{k} \frac{1-f_{k}}{1-e_{k}}=\prod_{k} \frac{\sin \pi \rho_{k}}{\sin \pi a_{k}} .
$$

The last relation is also implied from (1.1).

We remind that the monodromy group $G$ is reducible if and only if there exists a non-zero proper linear subspace $V$ in $C^{n}$ which is invariant under the action of $G$, and otherwise $G$ is irreducible. Let $\left\langle g_{1}, \cdots, g_{m}\right\rangle$ be the group generated by $g_{1}, \cdots, g_{m}$. Since $G=\left\langle M_{0}, M_{1}\right\rangle, G$ is reducible if and only if there exists $V\left(\neq\{0\}, C^{n}\right)$ with $V M_{\alpha} \subset V$ for $\alpha=0,1$.

THEOREM 2.1. If $f_{k}=1$ for some $k(1 \leq k \leq n)$, then $G$ is reducible (cf. (A) in $\left.\S 1\right)$.

ProOF. From (2.2) $\operatorname{rank}(\mathfrak{h})=n-1$. Let $W$ be a subspace of column vectors defined by $\left\{\boldsymbol{w} \in C^{n} ; \mathfrak{h} w=0\right\}$. Then $\operatorname{dim} W=1$ and, for any $\boldsymbol{w} \in W$, we can easily see $M_{\alpha} \boldsymbol{w}=\boldsymbol{w}$ $(\alpha=0,1)$. Let $V$ be the subspace of row vectors $v$ which satisfy $v \cdot w=0$ for any $w \in W$. Then $\operatorname{dim} V=n-1$ and $V M_{\alpha} \subset V(\alpha=0,1)$ follow.

q.e.d.

Let $E$ be the $n$ by $n$ diagonal matrix $\operatorname{diag}\left(e_{1}-1, \cdots, e_{n}-1\right)$. Then the next lemma also follows directly from (2.2).

LEMMA 2.2. If $f_{j} \neq 1$ for all $j(1 \leq j \leq n)$, then $\operatorname{det}(E \cdot \mathfrak{h}) \neq 0$.

THEOREM 2.3. Under the conditions $f_{j} \neq 1$, the following statements are equivalent:

(a) The monodromy group $G$ of $(\#)$ is irreducible.

(b) All the components $p_{j}$ and $q_{j}$ are non-zero.

(c) $e_{j} \neq f_{k}(j=1,2, \cdots, n-1 ; k=1,2, \cdots, n)$.

ProOF. The equivalence between (b) and (c) follows directly from (1.5).

(a) $\Rightarrow$ (c). It is sufficient to see the case where $e_{1}=f_{1}$. Then $p_{1}=0$ or $q_{1}=0$ by (1.6) and, consequently, 1-dimensional linear subspace $\left\{(*, 0, \cdots, 0) \in C^{n}\right\}$ or $(n-1)$-dimensional $\{(0, *, \cdots, *)\}$ is invariant under $G$, respectively. Similarly, if $e_{j}=f_{k}$, then $G$ is reducible.

(b) $\Rightarrow\left(\right.$ a). Let $V$ be a subspace invariant under $G$. For any $v \in V, v C_{\alpha}$ (see (1.3)) must be in $V$ because $v M_{\alpha}=v\left(I+C_{\alpha}\right)$ is in $V$. Let us denote the $k$-th row vector of $E \cdot h=C_{0}+C_{1}$ by $z_{k}$. Lemma 2.2 shows the linear independence of $z_{1}, \cdots, z_{n}$. For any $v=\left(v_{1}, \cdots, v_{n}\right) \in C^{n}$, the following relations hold: 


$$
\left\{\begin{array}{l}
v C_{0}=v_{1} 3_{1}+\cdots+v_{n-1} 3_{n-1}, \\
v C_{1}=v_{n} 3_{n} .
\end{array}\right.
$$

Now we consider two cases separately:

Case 1. There exists a vector $v \in V$ with $v_{n} \neq 0$.

Case 2. The $n$-th components of all vectors in $V$ are zero.

Case 1. For any $v$ with $v_{n} \neq 0, v C_{1}=v_{n 3_{n}} \in V$. Thus $z_{n}$ and, in particular, $3_{n} /\left(e_{n}-1\right)=\left(q_{1}, \cdots, q_{n-1}, 1\right)$ which we denote by $v_{0}$ are in $V$. By (2.3) we have $v_{0} C_{0}=q_{1} 3_{1}+\cdots+q_{n-1} 3_{n-1}=\left(\left(e_{1}-1\right) q_{1}, \cdots,\left(e_{n-1}-1\right) q_{n-1}, *\right) \in V$ and, consequently, $v_{0} C_{0} \cdot C_{0}=\left(e_{1}-1\right) q_{1} 3_{1}+\cdots+\left(e_{n-1}-1\right) q_{n-1} 3_{n-1}=\left(\left(e_{1}-1\right)^{2} q_{1}, \cdots,\left(e_{n-1}\right.\right.$ $\left.-1)^{2} q_{n-1}, *\right) \in V$ and so on. Repeating this process, we obtain that all vectors

$$
\left(e_{1}-1\right)^{k} q_{1} 3_{1}+\cdots+\left(e_{n-1}-1\right)^{k} q_{n-1} 3_{n-1} \quad(k=0,1, \cdots, n-2)
$$

in addition to $z_{n}$ are contained in $V$. Vandermond's determinant

$$
\operatorname{det}\left(\begin{array}{ccc}
q_{1} & \cdots & q_{n-1} \\
\left(e_{1}-1\right) q_{1} & \cdots & \left(e_{n-1}-1\right) q_{n-1} \\
\cdots \cdots \cdots \cdots \cdots \cdots \cdots \cdots \cdots \cdots \cdots \cdots \cdots \cdots \cdots \cdots \cdots \cdots \cdots & \cdots \cdots \cdots \cdots \cdots
\end{array}\right)=\prod_{i}^{\prime} q_{i} \cdot \prod_{j>k}\left(e_{j}-e_{k}\right)
$$

is non-zero from the assumptions (A) and $q_{j} \neq 0$, which leads to the linear independence of the above $n$ vectors. Thus $V=C^{n}$.

Case 2. For any $v=\left(v_{1}, \cdots, v_{n-1}, 0\right) \in V, v C_{0}=\left(v_{1}\left(e_{1}-1\right), \cdots, v_{n-1}\left(e_{n-1}-1\right)\right.$, $\left.\sum_{j}^{\prime}\left(e_{j}-1\right) v_{j} p_{j}\right)$ is in $V$, where the last component must be zero. Next $v C_{0} \cdot C_{0}=$ $\left(v_{1}\left(e_{1}-1\right)^{2}, \cdots, v_{n-1}\left(e_{n-1}-1\right)^{2}, \sum_{j}^{\prime}\left(e_{j}-1\right)^{2} v_{j} p_{j}\right)$ is in $V$ and also $\sum_{j}^{\prime}\left(e_{j}-1\right)^{2} v_{j} p_{j}=0$. Repeating it $n-1$ times, we obtain

$$
\sum_{j}^{\prime}\left(e_{j}-1\right)^{k} v_{j} p_{j}=0 \quad(k=1,2, \cdots, n-1) .
$$

As in the first case,

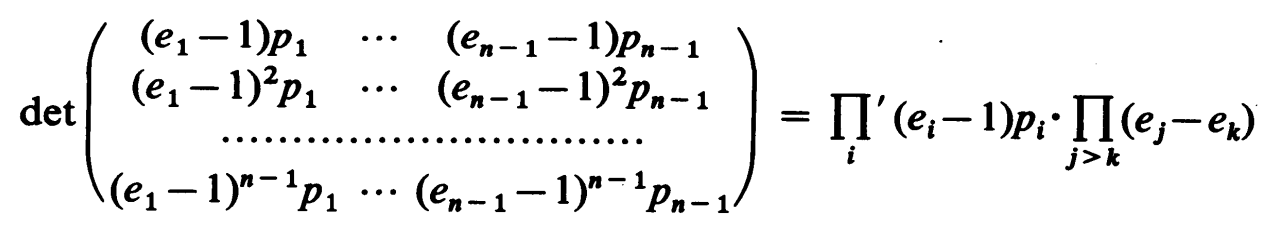

is not zero which leads $v_{j}=0$ for all $j(1 \leq j \leq n-1)$. Thus $v=0$ and, consequently, we have $V=\{\boldsymbol{0}\}$.

q.e.d.

We can restate Theorems 2.1 and 2.3 as follows:

THEOREM 2.4. The monodromy group $G$ of $(\#)$ is irreducible if and only if

$$
e_{j} \neq f_{k} \neq 1 \quad(j=1,2, \cdots, n-1 ; k=1,2, \cdots, n),
$$


i.e., none of the quantities $a_{j}-\rho_{k}$ and $\rho_{k}$ is an integer.

REMARK 2.5. The theorem was obtained independently in [1] (Propositions 2.7 and 3.3) by a method completely different from ours.

\section{§3. The invariant hermitian matrix.}

Definition 3.1. Let $\mathfrak{G}$ be a group in $G L(n, C)$. An $n$ by $n$ herimitian matrix $\mathfrak{S}$ is said to be $\mathfrak{F}$-invariant or invariant under $\mathfrak{S}$ if $g \mathfrak{S} g^{*}=\mathfrak{H}$ for any $g \in \mathfrak{F}$, where $g^{*}$ is the transposed complex conjugate of $g$.

Now we assume that all $p_{j} q_{j}$ are real numbers. It should be noticed that, if all $a_{j}$ and $\rho_{k}$ are real, this assumption is always satisfied by (1.6). Then we can define an $n$ by $n$ constant matrix $h$ as follows;

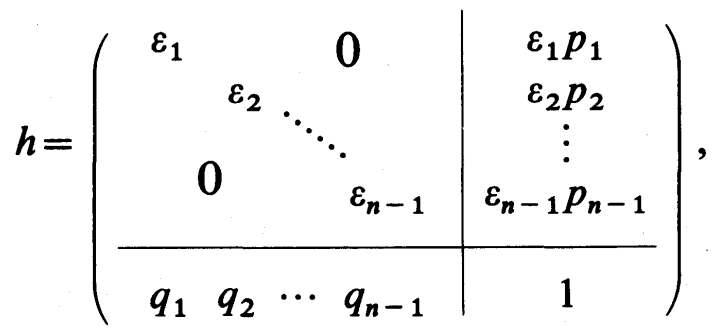

where each $\varepsilon_{j}$ is equal to 1 or -1 according to $p_{j} q_{j} \geq 0$ or $<0$, respectively. In particular, if $p_{j} q_{j}>0$ for all $j$, we have $h=\mathfrak{h}($ see $(2.1))$. Let $r=\#\left\{j ; p_{j} q_{j}<0\right\}$ for $j=1,2, \cdots, n-1$. Obviously we obtain

$$
\operatorname{det}(h)=(-1)^{r} \operatorname{det}(\mathfrak{h}) .
$$

When the inverse matrix of $h$ exists, we denote it by $H=h^{-1}$.

LEMMA 3.2. Suppose $f_{j} \neq 1$ for any $j=1,2, \cdots, n$ and $p_{k} q_{k}$ be real numbers for all $k=1,2, \cdots, n-1$. Then:

(a) $H$ exists.

(b) If $h$ is hermitian, then $H$ is a non-degenerate hermitian matrix invariant under $G$.

Proof. (a) Since $f_{j} \neq 1$, we have $\operatorname{det}(\mathfrak{h}) \neq 0$ by (2.2). Thus $H$ exists from (3.2).

(b) Non-degeneracy of $H$ follows immediately. The matrix $H$ is hermitian if and only if so is $h$. We show the $G$-invariance of $H$ as follows. Let $M_{0 j}=I+\left(e_{j}-1\right) Q_{j}$ $(j=1,2, \cdots, n-1)$ be matrices;

$$
I+\left(e_{j}-1\right)\left(\begin{array}{cccccc}
\frac{}{4} 0 \\
\hline 0 \cdots 0 & 1 & 0 & \cdots & 0 & p_{j} \\
\hline & 0 & &
\end{array}\right),
$$


where the $(j, j)$-th, the $(j, n)$-th and the other components of $Q_{j}$ are $1, p_{j}$ and 0 , respectively. Then we have $M_{0}=M_{01} M_{02} \cdots M_{0, n-1}$ and $M_{0 j} M_{0 k}=M_{0 k} M_{0 j}$ for any $j$ and $k$. Since $G=\left\langle M_{0}, M_{1}\right\rangle$, it is sufficient to show $M_{0 j} H M_{0 j}^{*}=H$ for all $j$ and $M_{1} H M_{1}^{*}=H$. By (3.3)

$$
M_{0 j} H M_{0 j}^{*}=H+\left(e_{j}-1\right) Q_{j} H+\left(\bar{e}_{j}-1\right) H Q_{j}^{*}+\left(e_{j}-1\right)\left(\bar{e}_{j}-1\right) Q_{j} H Q_{j}^{*},
$$

where $\bar{e}_{j}$ is the complex conjugate of $e_{j}$. Since $H=H^{*}$ and $H$ is the inverse of $h$ whose $j$-th row is identical with that of $Q_{j}$ up to $\varepsilon_{j}$, we have $Q_{j} H=H Q_{j}^{*}=\left(Q_{j} H\right)^{*}=\varepsilon_{j} E_{j j}$, where $E_{j j}$ is the matrix whose $(j, j)$-th component and the others are 1 and 0 , respectively. Moreover the only non-zero element of the $j$-th row of $Q_{j}^{*}$ is 1 . Thus the right hand side of (3.4) equals $H+\left[\left(e_{j}-1\right)+\left(\bar{e}_{j}-1\right)+\left(e_{j}-1\right)\left(\bar{e}_{j}-1\right)\right] \varepsilon_{j} E_{j j}=H$. The proof is the same for $M_{1}$.

q.e.d.

REMARK 3.3. In the case $h=\mathfrak{h}$, this lemma was proved by Okubo ([5], Chapter V).

By interchanging the order of the components $x_{1}, \cdots, x_{n-1}$ in (\#) if necessary, we may assume that $p_{j} q_{j}<0$ for $1 \leq j \leq r$ and $p_{j} q_{j} \geq 0$ for $r+1 \leq j \leq n-1$. If $1 \leq r \leq n-2$, the direct computation of $\operatorname{det}(\lambda I-h)$ shows that the eigenvalues of $h$ are $-1((r-1)$-ple), $1((n-r-2)-$ ple $)$ and the roots of algebraic equation

$$
\lambda^{3}-\lambda^{2}-\left(1-\sum_{j=1}^{r} p_{j} q_{j}+\sum_{j=r+1}^{n-1} p_{j} q_{j}\right) \lambda+\left(1-\sum_{j=1}^{n-1} p_{j} q_{j}\right)=0 \text {. }
$$

Similarly those for $r=0$ are $1\left((n-2)\right.$-ple) and the roots of $\lambda^{2}-2 \lambda+\operatorname{det}(\mathfrak{h})=0$, and, for $r=n-1,-1((n-2)-$ ple $)$ and the roots of $\lambda^{2}-\operatorname{det}(\mathfrak{h})=0$.

THEOREM 3.4. Under the same assumptions as in Lemma 3.2:

(a) If $0 \leq r \leq n-2$, then the signature $(p, q)$ of $H$ is equal to $(n-r, r)$ or $(n-r-1, r+1)$ according to $\operatorname{det}(\mathfrak{h})>0$ or $<0$, respectively.

(b) If $r=n-1$, then $\operatorname{det}(\mathfrak{h})>0$ and $(p, q)=(1, n-1)$.

Proof. If $\lambda$ is an eigenvalue of $h$, then $1 / \lambda$ is one of $H$. Let us denote the left hand side of $(3.5)$ by $f(\lambda)$. In case $1 \leq r \leq n-2$, we only note that $f(-1)=$ $2\left(1-\sum_{j=1}^{r} p_{j} q_{j}\right)>0, f(1)<0$ and $f(0)=\operatorname{det}(\mathfrak{h})$, and $f$ has a relative minimum and maximum at $\lambda_{+}>1$ and $\lambda_{-}<-1 / 3$, respectively. The assertion follows by an elementary consideration. The other cases are obvious.

q.e.d.

From now on we assume that the monodromy group $G$ of (\#) is irreducible and, all $a_{j}$ and $\rho_{k}$ are real numbers. Then, by (1.6) and Theorem 2.3, all $p_{j} q_{j}$ take real non-zero values. It was noticed in Remark 1.9 that, for investigating the group structure of $G$, it is sufficient to study $G^{\prime}=G\left(p_{j}^{\prime} ; q_{j}^{\prime}\right)=D^{-1} G D$ for an appropriate choice of a set $\left\{p_{j}^{\prime} ; q_{j}^{\prime}\right\}$ with $p_{j}^{\prime} q_{j}^{\prime}=p_{j} q_{j}$ by which the matrices $M_{\alpha}$ are transformed into suitable forms according to our purpose. We also note that the matrix (3.1), say $h^{\prime}$, determined by $G^{\prime}$ coincides with $D^{-1} h D$. From now on, if there is no danger of confusion, we denote $G^{\prime}, M_{\alpha}^{\prime}, p_{j}^{\prime}$, 
$q_{j}^{\prime}, h^{\prime}, \cdots$ again by $G, M_{\alpha}, p_{j}, q_{j}, h, \cdots$, respectively.

From the above argument and Lemma 3.2, we easily obtain:

THEOREM 3.5. Suppose $\left\{a_{j} ; \rho_{k}\right\}$ be a set of real numbers satisfying the assumption (A), the Riemann-Fuchs relation (1.1) and the conditions in Theorem 2.4. Then there exists an irreducible monodromy group $G$ of (\#) with parameters $\left\{a_{j}: \rho_{k}\right\}$ of which corresponding matrix $h$ defined by (3.1) is hermitian. And, consequently, $H=h^{-1}$ is a non-degenerate hermitian matrix invariant under $G$.

REMARK 3.6. Moreover, by the same reason as above, we can choose $G$ so that the corresponding $h$ is a real symmetric matrix.

Next we note that $M_{0}$ and $M_{1}$ are represented in terms of $e_{j}$ and $f_{k}$. On the other hand $2 n$ quantities $\left\{a_{j} ; \rho_{k}\right\}$ have only one relation (1.1). Moreover $a_{j}$ and $\rho_{k}$ are not integers from the assumption (A) and Theorem 2.4. Thus we may assume, without loss of generality,

$$
\left\{\begin{array}{l}
0<a_{1}<a_{2}<\cdots<a_{n-1}<1, \\
0<\rho_{1}<\rho_{2}<\cdots<\rho_{n}<1 .
\end{array}\right.
$$

THEOREM 3.7. Under the same assumptions as in Theorem 3.5 adding to (3.6), $H$ is a positive definite hermitian matrix if and only if the following condition holds;

$$
0<\rho_{1}<a_{1}<\rho_{2}<a_{2}<\cdots<a_{n-1}<\rho_{n}<1 \text { and } \rho_{1}<a_{n}<\rho_{n} .
$$

Proof. By Theorem 3.4, $H$ is positive definite if and only if $r=0$ and $\operatorname{det}(\mathfrak{h})>0$, namely, $p_{j} q_{j}>0$ for all $j$ and, under (3.6), $\sin \pi a_{n}>0$ from (2.2). Thus, by (1.6), we obtain the first relation in (3.7) which automatically leads to the second by the Riemann-Fuchs relation (1.1).

q.e.d.

REMARK 3.8. By Theorem 3.4 there are no cases where $H$ is negative definite.

\section{§4. The unitary reflection group $\boldsymbol{G}$ containing $\boldsymbol{G}$.}

We remind that $M_{0}$ is represented by the products $M_{01} M_{02} \cdots M_{0, n-1}(\operatorname{see}(3.3))$, where each $M_{0 j}$ is a so-called generalized reflection. Let us denote the group $\left\langle M_{01}, M_{02}, \cdots, M_{0, n-1}, M_{1}\right\rangle$ by $\tilde{G}$ which contains $G=\left\langle M_{0}, M_{1}\right\rangle$ as its subgroup. Note that the irreducibility of $G$ implies the same for $\tilde{G}$. Our purpose of this section is:

(*) Determine all cases where $\tilde{G}$ is a finite irreducible group for $n \geq 3$.

When $n=2$, it is equivalent to find all finite $G$ which was done by H. A. Schwarz in his famous paper [9]. Suppose $\tilde{G}$ is finite. Then $G$ is also finite and, consequently, all solutions of (\#) are algebraic functions. Thus all quantities $a_{j}$ and $\rho_{j}(j=1,2, \cdots, n)$ are rational numbers. It should also be noticed that $\tilde{G}$ must be isomorphic to a finite group generated by $n$ unitary reflections. All such groups were classified by 
Shephard-Todd [10]. We denote by $S T k$ the number $k(1 \leq k \leq 37)$ of the group on the table VII in [10].

LEMMA 4.1. Suppose $\tilde{G}$ be a finite irreducible group. Then it is isomorphic to one of $S T 25, S T 26$ and $G(m, 1,3)(m \geq 3)$.

ProOf. Since none of $a_{j}-a_{k}(j, k=1, \cdots, n-1)$ is an integer by the assumption (A), two of $a_{1}, \cdots, a_{n-1}$ cannot be equal to $1 / 2$ simultaneously. Thus $\tilde{G}$ is finite only in the following four cases; $S T 25, S T 26$ and $S T 32$ for primitive $\tilde{G}$ from the table VIII in [10], and $G(m, 1,3)(m \geq 3)$ for imprimitive $\tilde{G}$. But, for $S T 32(n=4), a_{1}, a_{2}$ and $a_{3}$ must be equal to $1 / 3$ or $2 / 3$ which is impossible from the same reason as above. Lemma follows.

q.e.d.

Since $n=3$ in all the possible cases, we restate several acquired results for the later use. The relations (1.1) and (1.6) are as follows:

$$
\begin{gathered}
a_{1}+a_{2}+a_{3}=\rho_{1}+\rho_{2}+\rho_{3} . \\
\left\{\begin{array}{l}
p_{1} q_{1}=-\frac{\sin \pi\left(a_{1}-\rho_{1}\right) \cdot \sin \pi\left(a_{1}-\rho_{2}\right) \cdot \sin \pi\left(a_{1}-\rho_{3}\right)}{\sin \pi a_{1} \cdot \sin \pi a_{3} \cdot \sin \pi\left(a_{1}-a_{2}\right)} \\
p_{2} q_{2}=-\frac{\sin \pi\left(a_{2}-\rho_{1}\right) \cdot \sin \pi\left(a_{2}-\rho_{2}\right) \cdot \sin \pi\left(a_{2}-\rho_{3}\right)}{\sin \pi a_{2} \cdot \sin \pi a_{3} \cdot \sin \pi\left(a_{2}-a_{1}\right)}
\end{array}\right.
\end{gathered}
$$

In the proof of Lemma 3.2 we have already shown that $H$ is also $\tilde{G}$-invariant. Therefore, by Theorem 3.7 , the $G$ - and $\tilde{G}$-invariant hermitian matrix $H$ is positive definite if and only if

$$
0<\rho_{1}<a_{1}<\rho_{2}<a_{2}<\rho_{3}<1 \text { and } 0<\rho_{1}<a_{3}<\rho_{3}<1 \text {. }
$$

Moreover, by Remark 1.9, we take $G\left(p_{j} q_{j} ; 1\right)$ for simplifying our later computations;

$$
\begin{cases}M_{01} & =\left(\begin{array}{ccc}
e_{1} & 0 & \left(e_{1}-1\right) p_{1} q_{1} \\
0 & 1 & 0 \\
0 & 0 & 1
\end{array}\right), \\
M_{1}=\left(\begin{array}{ccc}
1 & 0 & 0 \\
0 & 1 & 0 \\
e_{3}-1 & e_{3}-1 & e_{3}
\end{array}\right) .\end{cases}
$$

The next theorem due to T. A. Springer ([11], 3.4) plays an important role for our following investigation. Let $\boldsymbol{G}$ be a finite complex reflection group and $\zeta$ a root of unity, of order $d$. We denote the degrees of $\left(5\right.$ by $d_{i}(i=1,2, \cdots, n)$ (cf. [10], Table VII).

THEOREM 4.2 (Springer [11]). There exists $g \in(\mathfrak{S}$ with eigenvalue $\zeta$ if and only if $d$ divides at least one degree $d_{i}$. 
By virtue of these results we determine finite $\tilde{G}$ up to the complex conjugate. We state our process of determinations explicitly only for the case $\widetilde{G} \simeq S T 25$.

[ST 25]. By the Table VIII in [10], each $a_{j}$ is equal to either $1 / 3$ or $2 / 3$, and, consequently, $a_{1}=1 / 3$ and $a_{2}=2 / 3$ from (4.3). It is sufficient to study the case $\left(a_{1}, a_{2}, a_{3}\right)=(1 / 3,2 / 3,1 / 3)$, for the remaining $(1 / 3,2 / 3,2 / 3)$ is its complex conjugate. Set $\rho_{j}=c_{j} / d_{j},\left(c_{j}, d_{j}\right)=1$. Since the degrees of $S T 25$ are $\{6,9,12\}$ and $\rho_{j}$ are eigenvalues of $M_{\infty}, d_{j}$ must be a divisor of 9 or 12 by Theorem 4.2 . We first take all such possible $\rho_{2}$ satisfying $1 / 3<\rho_{2}<2 / 3$. Then $\rho_{2}$ is equal to one of $\{5 / 12,4 / 9,1 / 2,5 / 9,7 / 12\}$. We note that, for carrying out this and the following processes, it is convenient to consider $\rho_{j}$ in the form $c_{j}^{\prime} / 36$, where $c_{j}^{\prime}$ has 3 or 4 as its divisor.

Next, for each $\rho_{2}$ stated above, we seek all possible pairs of $\rho_{1}$ and $\rho_{3}$ satisfying (4.1) and (4.3). Then there are only four cases;

$$
\begin{aligned}
\left(a_{j} ; \rho_{j}\right)= & \left(a_{1}, a_{2}, a_{3} ; \rho_{1}, \rho_{2}, \rho_{3}\right) \\
= & \left(\frac{1}{3}, \frac{2}{3}, \frac{1}{3} ; \frac{1}{12}, \frac{5}{12}, \frac{5}{6}\right), \quad\left(\frac{1}{3}, \frac{2}{3}, \frac{1}{3} ; \frac{1}{6}, \frac{5}{12}, \frac{3}{4}\right), \\
& \left(\frac{1}{3}, \frac{2}{3}, \frac{1}{3} ; \frac{1}{9}, \frac{4}{9}, \frac{7}{9}\right), \quad\left(\frac{1}{3}, \frac{2}{3}, \frac{1}{3} ; \frac{1}{12}, \frac{1}{2}, \frac{3}{4}\right) .
\end{aligned}
$$

For each case, by substituting those values into (4.2), we obtain $M_{0 j}$ and $M_{1}$ from (4.4). Again by the Table VIII in [10], if $\tilde{G}$ is isomorphic to $S T 25$, then the following conditions should be satisfied;

(4.6) the orders of $M_{0}=M_{01} M_{02}, M_{01} M_{1}$ and $M_{02} M_{1}$ are equal to 2, 3, 4, or 6 .

Since

$$
M_{0}{ }^{n}=\left(\begin{array}{ccc}
e_{1}{ }^{n} & 0 & \left(e_{1}{ }^{n}-1\right) p_{1} q_{1} \\
0 & e_{2}{ }^{n} & \left(e_{2}{ }^{n}-1\right) p_{2} q_{2} \\
0 & 0 & 1
\end{array}\right)
$$

$M_{0}{ }^{3}=I$ holds in any of (4.5). We checked by using MACSYMA that, for each case, whether the conditions (4.6) for the remaining two matrices are satisfied or not. Then they are fulfilled only for the third case in (4.5). In the other cases, $\left(M_{01} M_{1}\right)^{n} \neq I$ and $\left(M_{02} M_{1}\right)^{n} \neq I$ for any $n=2,3,4,6$.

For $\left(a_{j} ; \rho_{j}\right)=(1 / 3,2 / 3,1 / 3 ; 1 / 9,4 / 9,7 / 9)$, we have

$$
M_{01}=\left(\begin{array}{ccc}
\omega & 0 & (\omega-1) / 3 \\
0 & 1 & 0 \\
0 & 0 & 1
\end{array}\right), \quad M_{02}=\left(\begin{array}{ccc}
1 & 0 & 0 \\
0 & \omega^{2} & \left(\omega^{2}-1\right) / 3 \\
0 & 0 & 1
\end{array}\right),
$$




$$
M_{1}=\left(\begin{array}{ccc}
1 & 0 & 0 \\
0 & 1 & 0 \\
\omega-1 & \omega-1 & \omega
\end{array}\right)
$$

where $\omega=\exp (2 \pi \sqrt{-1} / 3)$, and $\left(M_{01} M_{1}\right)^{6}=\left(M_{02} M_{1}\right)^{4}=I$. Let $T$ be a constant matrix;

$$
T=\left(\begin{array}{ccc}
1 & 0 & -1 \\
0 & 1 & -1 \\
0 & 0 & 3
\end{array}\right)
$$

Then we have

$$
\begin{gathered}
T^{-1} M_{01} T=\left(\begin{array}{ccc}
\omega & 0 & 0 \\
0 & 1 & 0 \\
0 & 0 & 1
\end{array}\right), \quad T^{-1} M_{02} T=\left(\begin{array}{ccc}
1 & 0 & 0 \\
0 & \omega^{2} & 0 \\
0 & 0 & 1
\end{array}\right), \\
T^{-1} M_{1} T=-\frac{\sqrt{-1}}{\sqrt{3}}\left(\begin{array}{ccc}
\omega & \omega^{2} & \omega^{2} \\
\omega^{2} & \omega & \omega^{2} \\
\omega^{2} & \omega^{2} & \omega
\end{array}\right),
\end{gathered}
$$

which are well-known generators of $S T 25$ (see [10], p. 296). Thus $\tilde{G}$ is just the group $S T 25$. Moreover, taking a matrix $S$,

$$
S=\frac{1}{\sqrt{3}}\left(\begin{array}{ccc}
\omega & 1 & \omega^{2} \\
\omega & \omega^{2} & 1 \\
\omega & \omega & \omega
\end{array}\right)
$$

we obtain

$$
(T S)^{-1} M_{0}(T S)=\left(\begin{array}{lll}
0 & 1 & 0 \\
0 & 0 & 1 \\
1 & 0 & 0
\end{array}\right), \quad(T S)^{-1} M_{1}(T S)=\left(\begin{array}{ccc}
\omega & 0 & 0 \\
0 & 1 & 0 \\
0 & 0 & 1
\end{array}\right) .
$$

It shows that $G \simeq((Z / 3 Z) \times(Z / 3 Z) \times(Z / 3 Z)) \triangleleft(Z / 3 Z)$ and is contained in $G(3,1,3)$. We note that $G$ is an imprimitive group, though $\tilde{G}$ is primitive.

[ST 26] and $G(3,1,3)$. In these cases the order of each reflection is equal to 2 or 3. If $\tilde{G}$ is isomorphic to one of these groups, there necessarily exists at least one reflection of each order among $M_{01}, M_{02}$ and $M_{1}$ (see [10], p. 295 and the Table VIII). Then there are four possible cases on the choice of $a_{j}$ 's up to complex conjugates;

$$
\left(a_{1}, a_{2}, a_{3}\right)=\left(\frac{1}{3}, \frac{2}{3}, \frac{1}{2}\right),\left(\frac{1}{3}, \frac{1}{2}, \frac{1}{3}\right),\left(\frac{1}{2}, \frac{2}{3}, \frac{1}{3}\right),\left(\frac{1}{3}, \frac{1}{2}, \frac{1}{2}\right) .
$$


The degrees of $S T 26$ and $G(3,1,3)$ are $\{9,12,18\}$ and $\{3,6,9\}$, respectively. Similar arguments to [ST25] show that there are 47 possible cases which we have to examine. On the other hand, if $\widetilde{G}$ is actually isomorphic to the group $S T 26$ or $G(3,1,3)$, then the same condition as in (4.6) should also be satisfied. Performing the same calculation as in [ST 25] by using MACSYMA, we can see that there are only three possible cases;

$$
\begin{aligned}
\left(a_{j} ; \rho_{j}\right)= & \left(\frac{1}{3}, \frac{1}{2}, \frac{1}{3} ; \frac{1}{18}, \frac{7}{18}, \frac{13}{18}\right), \\
& \left(\frac{1}{2}, \frac{2}{3}, \frac{1}{3} ; \frac{1}{12}, \frac{7}{12}, \frac{5}{6}\right), \\
& \left(\frac{1}{3}, \frac{1}{2}, \frac{1}{2} ; \frac{1}{9}, \frac{4}{9}, \frac{7}{9}\right) .
\end{aligned}
$$

The case (4.7) $)_{1}$ : By substituting (4.7) $)_{1}$ into (4.4), we obtain

$$
\begin{gathered}
M_{01}=\left(\begin{array}{ccc}
\omega & 0 & (\omega-1) / 3 \\
0 & 1 & 0 \\
0 & 0 & 1
\end{array}\right), M_{02}=\left(\begin{array}{ccc}
1 & 0 & 0 \\
0 & -1 & -1 \\
0 & 0 & 1
\end{array}\right), \quad M_{1}=\left(\begin{array}{cccc}
1 & 0 & 0 \\
0 & 1 & 0 \\
\omega-1 & \omega-1 & \omega
\end{array}\right), \\
M_{0}{ }^{6}=\left(M_{01} M_{1}\right)^{6}=\left(M_{02} M_{1}\right)^{6}=I .
\end{gathered}
$$

Taking $T=\left(\begin{array}{ccc}1 & 0 & 0 \\ 1 & -1 & 0 \\ -2 & 1 & 1\end{array}\right)$, we have

$$
\left\{\begin{array}{l}
T^{-1} M_{01} T=-\frac{\sqrt{-1}}{\sqrt{3}}\left(\begin{array}{ccc}
\omega & \omega^{2} & \omega^{2} \\
\omega^{2} & \omega & \omega^{2} \\
\omega^{2} & \omega^{2} & \omega
\end{array}\right), \quad T^{-1} M_{02} T=\left(\begin{array}{ccc}
1 & \\
& 1 \\
& 1
\end{array}\right) \\
T^{-1} M_{1} T=\left(\begin{array}{ccc}
1 & \\
& 1 & \\
& & \omega^{2}
\end{array}\right)
\end{array}\right.
$$

The case $(4.7)_{2}$ :

$$
\begin{gathered}
M_{01}=\left(\begin{array}{ccc}
-1 & 0 & -1 \\
0 & 1 & 0 \\
0 & 0 & 1
\end{array}\right), M_{02}=\left(\begin{array}{ccc}
1 & 0 & 0 \\
0 & \omega^{2} & \left(\omega^{2}-1\right) / 3 \\
0 & 0 & 1
\end{array}\right), M_{1}=\left(\begin{array}{ccc}
1 & 0 & 0 \\
0 & 1 & 0 \\
\omega-1 & \omega-1 & \omega
\end{array}\right) . \\
M_{0}{ }^{6}=\left(M_{01} M_{1}\right)^{6}=\left(M_{02} M_{1}\right)^{4}=I .
\end{gathered}
$$


If we take $T=\left(\begin{array}{ccc}-1 & 1 & 0 \\ 0 & 1 & 0 \\ 1 & -2 & 1\end{array}\right)$, then

$$
\left\{\begin{array}{l}
T^{-1} M_{01} T=\left(\begin{array}{lll} 
& & 1 \\
& 1 & \\
1 & &
\end{array}\right), \quad T^{-1} M_{02} T=\frac{\sqrt{-1}}{\sqrt{3}}\left(\begin{array}{ccc}
\omega^{2} & \omega & \omega \\
\omega & \omega^{2} & \omega \\
\omega & \omega & \omega^{2}
\end{array}\right), \\
T^{-1} M_{1} T=\left(\begin{array}{lll}
1 & & \\
& 1 & \\
& &
\end{array}\right) .
\end{array}\right.
$$

Thus, in both cases, $\tilde{G}$ is just the group $S T 26$ from (4.8), (4.9) and [10], p. 297. We easily see that, in each case, $\left\langle M_{01}, M_{02}\right\rangle=\left\langle M_{0}\right\rangle$ from (4.8) and (4.9). Thus $G=\tilde{G}=$ $S T 26$ in both cases $(4.7)_{1}$ and (4.7) 2 .

The case $(4.7)_{3}$ :

$$
\begin{gathered}
M_{01}=\left(\begin{array}{ccc}
\omega & 0 & (\omega-1) / 2 \\
0 & 1 & 0 \\
0 & 0 & 1
\end{array}\right), M_{02}=\left(\begin{array}{ccc}
1 & 0 & 0 \\
0 & -1 & -1 / 2 \\
0 & 0 & 1
\end{array}\right), M_{1}=\left(\begin{array}{ccc}
1 & 0 & 0 \\
0 & 1 & 0 \\
-2 & -2 & -1
\end{array}\right) . \\
M^{6}=\left(M_{01} M_{1}\right)^{6}=\left(M_{02} M_{1}\right)^{3}=I .
\end{gathered}
$$

If we take

$$
S=\left(\begin{array}{ccc}
1 & 1 & 1 \\
0 & 1 & 0 \\
0 & -2 & -2
\end{array}\right)
$$

then we have

$$
S^{-1} M_{01} S=\left(\begin{array}{ccc}
\omega & & \\
& 1 & \\
& & 1
\end{array}\right), S^{-1} M_{02} S=\left(\begin{array}{lll}
1 & & \\
& & 1 \\
& 1
\end{array}\right), \quad S^{-1} M_{1} S=\left(\begin{array}{lll} 
& & 1 \\
& 1 & \\
1 & &
\end{array}\right) .
$$

Thus $\tilde{G}=G(3,1,3)$. Since $\left\langle M_{01}, M_{02}\right\rangle=\left\langle M_{0}\right\rangle$, we obtain $G=\tilde{G}=G(3,1,3)$.

$G(m, 1,3)(m \geq 4)$. In [8] we found more general class of imprimitive groups on the analogy of the last case. Let us consider the case where

$$
\left(a_{j} ; \rho_{j}\right)=\left(\frac{n}{m}, \frac{1}{2}, \frac{1}{2} ; \frac{n}{3 m}, \frac{n+m}{3 m}, \frac{n+2 m}{3 m}\right),
$$


for any positive integer $n$ satisfying $(m, n)=1$ and $1 \leq n<m / 2$. Then we have

$$
\begin{gathered}
M_{01}=\left(\begin{array}{ccc}
\zeta_{m}{ }^{n} & 0 & \left(\zeta_{m}{ }^{n}-1\right) / 2 \\
0 & 1 & 0 \\
0 & 0 & 1
\end{array}\right), \quad M_{02}=\left(\begin{array}{ccc}
1 & 0 & 0 \\
0 & -1 & -1 / 2 \\
0 & 0 & 1
\end{array}\right) \\
M_{1}=\left(\begin{array}{ccc}
1 & 0 & 0 \\
0 & 1 & 0 \\
-2 & -2 & -1
\end{array}\right) .
\end{gathered}
$$

Taking the same matrix $S$ as in (4.10), we obtain

$$
S^{-1} M_{01} S=\left(\begin{array}{ccc}
\zeta_{n}{ }^{m} & & \\
& 1 & \\
& & 1
\end{array}\right), \quad S^{-1} M_{02} S=\left(\begin{array}{lll}
1 & & \\
& & 1 \\
& 1 &
\end{array}\right), \quad S^{-1} M_{1} S=\left(\begin{array}{lll} 
& 1 \\
& 1 & \\
1 & &
\end{array}\right) .
$$

Thus $\tilde{G}$ is certainly the group $G(m, 1,3)$. If $m$ is odd, then $\left\langle M_{01}, M_{02}\right\rangle=\left\langle M_{0}\right\rangle$ and, consequently, $G=\widetilde{G}=G(m, 1,3)$. However, if $m$ is even, we easily obtain $\left\langle M_{01}, M_{02}\right\rangle \supsetneq\left\langle M_{0}\right\rangle$ and $G \simeq\left\langle G(m / 2,1,3), \zeta_{m} I\right\rangle$ by an elementary calculation. The case $(4.7)_{3}$ is a special case of (4.11). We note that, if $m / 2<n<m$ in (4.11), $\widetilde{G}$ (and also $G$ ) is the complex conjugate to the group determined by

$$
\left(\frac{m-n}{m}, \frac{1}{2}, \frac{1}{2} ; \frac{m-n}{3 m}, \frac{(m-n)+m}{3 m}, \frac{(m-n)+2 m}{3 m}\right) \text {. }
$$

Moreover, if $\tilde{G}$ and $G$ correspond to a parameter set $\left(a_{j} ; \rho_{j}\right)$, their complex conjugates are given by $\left(1-a_{j} ; 1-\rho_{j}\right)$.

Now we summarize our results in a single theorem.

THEOREM 4.3. Suppose the monodromy group $G$ of (\#) is irreducible. Then;

(a) $\tilde{G}$ is a finite primitive group if and only if $\left(a_{j} ; \rho_{j}\right)$ takes one of the following values;

( I ) $\left(\mathfrak{l} \pm \frac{1}{3}, \mathfrak{m} \pm \frac{2}{3}, \mathfrak{n} \pm \frac{1}{3} ; \mathfrak{r} \pm \frac{1}{9}, \mathfrak{s} \pm \frac{4}{9}, \mathfrak{t} \pm \frac{7}{9}\right)$,

(II) $\left(\mathrm{I} \pm \frac{1}{3}, \mathfrak{m} \pm \frac{1}{2}, \mathfrak{n} \pm \frac{1}{3} ; \mathfrak{r} \pm \frac{1}{18}, \mathfrak{s} \pm \frac{7}{18}, \mathfrak{t} \pm \frac{13}{18}\right)$,

(III) $\left(\mathrm{l} \pm \frac{1}{2}, \mathrm{~m} \pm \frac{2}{3}, \mathrm{n} \pm \frac{1}{3} ; \mathfrak{r} \pm \frac{1}{12}, \mathfrak{s} \pm \frac{7}{17}, \mathfrak{t} \pm \frac{5}{6}\right)$

where $\mathfrak{l}, \mathfrak{m}, \mathfrak{n}, \mathfrak{r}, \mathfrak{s}, \mathfrak{t} \in Z, \mathfrak{n}=\mathfrak{r}+\mathfrak{s}+\mathfrak{t}-\mathfrak{l}-\mathfrak{m}$ and the sign \pm takes either + simultaneously or - simultaneously. In each case the following properties are satisfied; 


\begin{tabular}{cccccccc}
\hline & \multirow{2}{*}{$\boldsymbol{G}$} & \multirow{6}{*}{$\boldsymbol{G}$} & \multicolumn{6}{c}{ order of } & \multirow{2}{*}{$g$} \\
\cline { 3 - 7 } & & & $G$ & $M_{0}$ & $M_{1}$ & $M_{\infty}$ & \\
\hline (I ) & $S T 25$ & $\left(Z_{3} \times Z_{3} \times Z_{3}\right) \rtimes Z_{3}$ & 81 & 3 & 3 & 9 & 10 \\
(II) & $S T 26$ & $S T 26$ & 1296 & 6 & 3 & 18 & $17^{2}$ \\
(III) & $S T 26$ & $S T 26$ & 1296 & 6 & 3 & 12 & 271 \\
\hline
\end{tabular}

where $g$ is the genus of the Riemann surface of the solutions for (\#).

(b) Suppose $m \geq 3$. $\tilde{G}$ is a finite imprimitive group if

$$
\left(a_{j} ; \rho_{j}\right)=\left(\mathfrak{l} \pm \frac{n}{m}, \mathfrak{m} \pm \frac{1}{2}, \mathfrak{n} \pm \frac{1}{2} ; \mathfrak{r} \pm \frac{n}{3 m}, \mathfrak{s} \pm \frac{m+n}{3 m}, \mathfrak{t} \pm \frac{3 m+n}{3 m}\right)
$$

for any positive integer $n$ satisfying $(m, n)=1$ and $1 \leq n \leq m$. The following is similar to (a);

$\frac{m ; \text { odd }}{m ; \text { even }} G(m, 1,3) \frac{G(m, 1,3)}{\left\langle G(m / 2,1,3), \zeta_{m} I\right\rangle} \frac{6 m^{3}}{3 m^{3} / 2} \frac{2 m}{m} \frac{2}{2} \frac{3 m}{3 m} \frac{(m-1)\left(3 m^{2}-2 m-2\right) / 2}{(m-2)\left(3 m^{2}-2 m-4\right) / 8}$

Proof. It is easy to obtain the orders of $G$ from the Table VII in [10]. We only note that, for even $m$ in (b), $G(m, 1,3) / G(m / 2,1,3) \simeq Z / 2 Z$. Let us denote the order of $M_{\alpha}$ by $\mathfrak{D}_{\alpha}(\alpha=0,1, \infty)$. The genus in each case is led from the orders $|G|$ and $\mathfrak{o}_{\alpha}$ by using the Hurwitz formula.

$$
g=1-|G|+\frac{|G|}{2}\left\{3-\left(1 / \mathrm{o}_{0}\right)-\left(1 / \mathrm{o}_{1}\right)-\left(1 / \mathrm{o}_{\infty}\right)\right\}
$$

q.e.d.

REMARK 4.4. Now we have to mention the relation between $G$ and the group $H(a ; b)$ in [1]. We use the notations in [1] freely. For the classical generalized hypergeometric equation (b) in $\S 1$, one of $b_{j}$ 's is equal to 1 (say, $b_{n}=1$ ). Since $\operatorname{det}\left(\lambda I-M_{0}\right)=(\lambda-1) \prod_{j}^{\prime}\left(\lambda-e_{j}\right)=(\lambda-1) \prod_{j}^{\prime}\left(\lambda-b_{j}^{-1}\right)=\operatorname{det}\left(\lambda I-h_{0}\right)$ and $\operatorname{det}(\lambda I-$ $\left.M_{\infty}\right)=\prod_{j}\left(\lambda-f_{j}\right)=\prod_{j}\left(\lambda-a_{j}^{-1}\right)=\operatorname{det}\left(\lambda I-h_{\infty}^{-1}\right)$ with $M_{1} M_{0}=M_{\infty}$ and $h_{\infty} h_{1} h_{0}=I, G$ is just the hypergeometric group defined in Definition 3.1 in [1]. Thus, by Levelt's theorem ([1], Theorem 3.5), we have:

LEMMA 4.5. $G$ is conjugate to $H\left(f_{1}^{-1}, \cdots, f_{n}^{-1} ; e_{1}^{-1}, \cdots, e_{n-1}^{-1}, 1\right)$ in $G L(n, C)$.

We point out the following two facts. First, as was mentioned above, the case (IV) has been obtained intuitively on the analogy of $G(3,1,3)$. We checked by using 
MACSYMA that it is the only case for $\tilde{G}$ to be finite imprimitive when $3 \leq m \leq 6$, and we easily see from Theorem 5.8 in [1] that the same is true for any $m \geq 3$. Note also that, in the case (I), the reflection subgroup $H_{r}$ of $H$ acts reducibly on $C^{3}$ ([1], Theorem 5.3) and, the cases (II) and (III) are (1/2)-shift of No. 10 and (1/6)-shift of No. 9 in the Table 8.3 in [1], respectively.

Second, to obtain the key result stated in Theorem 4.8 in [1], it is important that, under a suitable condition, $H$ is contained in $G L\left(n, \mathcal{O}_{K}\right)$, where $\mathcal{O}_{K}$ is the ring of integers of a cyclotomic field $K$. Moreover, in each of our cases stated in Theorem 4.3, we also have $\widetilde{G} \subset G L\left(n, \mathcal{O}_{K}\right)$ for a suitable $K$. Note that:

PROPOSITION 4.6. Let $G$ be an irreducible group with an invariant hermitian matrix $H$ which is also invariant under $\tilde{G}$. Let $K / Q$ be a finite Galois extension of which subfield $K \cap \boldsymbol{R}$ is also a Galois extension over $Q$ and let $X^{-1} \tilde{G} X \subset G L\left(n, \mathcal{O}_{K}\right)$ for some $X \in G L(n, C)$. Then $\tilde{G}$ is finite if and only if so is $G$.

On the other hand our result shows that, for the cases which are listed on the Table 8.3 in [1] satisfying either $\alpha_{1}=0$ or $\beta_{1}=0$, the corresponding $\tilde{G}$ are all infinite groups. For several (probably, for all) cases, we can easily check that the trace of $M_{0 j} M_{j}$ is not an algebraic integer.

\section{References}

[ 1 ] F. BeuKers and G. HeCKman, Monodromy for the hypergeometric function ${ }_{n} F_{n-1}$, Invent. Math., 95 (1989), 325-354.

[2] A. ERDÉLYI, Higher Transcendental Functions, Vol I, McGraw-Hill, 1953.

[3] M. Iwano, Schwarz Theory, Math. Sem. Rep., Tokyo Metro. Univ., 1989.

[4] A. H. M. LeVelt, Hypergeometric Functions, Amsterdam, 1961.

[5] K. OKuBo, On the Group of Fuchsian Equations, Math. Sem. Rep.,'Tokyo Metro. Univ., 1987.

[6] K. OKUBO and K. TAKANO, Generalized hypergeometric functions, preprint, 1981.

[7] K. OKubo, K. TaKano and S. Yoshida, A connection problem for the generalized hypergeometric equation, Funkcial. Ekvac., 31 (1988), 483-495.

[8] T. SASAI, (a) Generalized hypergeometric equations with finite monodromy groups (in Japanese), RIMS Kokyuroku, Kyoto Univ., 681 (1989), 123-139, (b) Generalized hypergeometric equations with certain finite irreducible monodromy groups, Proc. Japan Acad., 65 (1989), 223-226.

[9] H. A. Schwarz, Über diejenigen Fälle, in welchen die Gaussische hypergeometrische Reihe eine algebraische Funktion ihres vierten Elementes darstellt, J. Reine Angew. Math., 75 (1873), 292-335.

[10] G. C. SHEPHARD and J. A. TodD, Finite unitary reflection groups, Canad. J. Math., 6 (1954), 274 304.

[11] T. A. SPRINGER, Regular elements of finite reflection groups, Invent. Math., 25 (1974), 159-198.

[12] K. TAKano and E. BANNAI, A global study of Jordan-Pochhammer differential equations, Funkcial. Ekvac., 19 (1976), 85-99.

[13] M. YoshidA, Fuchsian Differential Equations, Vieveg, 1987.

Present Address:

Department of Mathematics, Tokyo Metropolitan University

Minami-Ohsawa, Hachioji-ShI, ToKyo 192-03, JAPaN 